\title{
BMJ Global Health A network meta-analysis of psychosocial interventions for refugees and asylum seekers with PTSD
}

Giulia Turrini, ${ }^{1}$ Federico Tedeschi, ${ }^{1}$ Pim Cuijpers, ${ }^{2}$ Cinzia Del Giovane, ${ }^{3}$ Ahlke Kip, ${ }^{4}$ Nexhmedin Morina, ${ }^{4}$ Michela Nosè, ${ }^{1}$ Giovanni Ostuzzi, ${ }^{1}$ Marianna Purgato, ${ }^{1}$ Chiara Ricciardi, ${ }^{1}$ Marit Sijbrandij, ${ }^{2}$ Wietse Tol, ${ }^{5,6,7}$ Corrado Barbui ${ }^{1}$

To cite: Turrini G, Tedeschi F, Cuijpers $\mathrm{P}$, et al. A network meta-analysis of psychosocial interventions for refugees and asylum seekers with PTSD. BMJ Global Health 2021;6:e005029. doi:10.1136/ bmjgh-2021-005029

Handling editor Soumyadeep Bhaumik

- Additional supplemental material is published online only. To view, please visit the journal online (http://dx.doi.org/10. 1136/bmjgh-2021-005029)

Received 18 January 2021 Accepted 24 May 2021

Check for updates

C Author(s) (or their employer(s)) 2021. Re-use permitted under CC BY-NC. No commercial re-use. See rights and permissions. Published by BMJ.

For numbered affiliations see end of article.

Correspondence to Dr Giulia Turrini; giulia.turrini@univr.it

\section{ABSTRACT}

Introduction Refugees and asylum seekers are vulnerable to common mental disorders, including post-traumatic stress disorder (PTSD). Using a network meta-analysis (NMA) approach, the present systematic review compared and ranked psychosocial interventions for the treatment of PTSD in adult refugees and asylum seekers.

Methods Randomised studies of psychosocial interventions for adult refugees and asylum seekers with PTSD were systematically identified. PTSD symptoms at postintervention was the primary outcome. Standardised mean differences (SMDs) and ORs were pooled using pairwise and NMA. Study quality was assessed with the Cochrane Risk of Bias ( $\mathrm{RoB})$ tool, and certainty of evidence was assessed through the Confidence in Network MetaAnalysis application.

Results A total of 23 studies with 2308 participants were included. Sixteen studies were conducted in high-income countries, and seven in low-income or middle-income countries. Most studies were at low risk of bias according to the Cochrane RoB tool. NMA on PTSD symptoms showed that cognitive behavioural therapy (CBT) $(\mathrm{SMD}=-1.41 ; 95 \% \mathrm{Cl}-2.43$ to -0.38$)$ and eye movement desensitisation and reprocessing (EMDR) (SMD $=-1.30$; $95 \% \mathrm{Cl}-2.40$ to -0.20 ) were significantly more effective than waitlist (WL). CBT was also associated with a higher decrease in PTSD symptoms than treatment as usual (TAU) (SMD -1.51; 95\% Cl -2.67 to -0.36). For all other interventions, the difference with WL and TAU was not significant. CBT and EMDR ranked best according to the mean surface under the cumulative ranking. Regarding acceptability, no intervention had less dropouts than inactive interventions

Conclusion CBT and EMDR appeared to have the greatest effects in reducing PTSD symptoms in asylum seekers and refugees. This evidence should be considered in guidelines and implementation packages to facilitate dissemination and uptake in refugee settings.

\section{INTRODUCTION}

The right of everyone to enjoy the highest attainable standard of physical and mental health is established in the WHO Constitution of 1946. International human rights standards

\section{Key questions}

What is already known?

- Research suggests that psychosocial interventions are effective in treating post-traumatic stress disorder (PTSD) and related symptoms in individuals who were exposed to traumatic events.

- Existing systematic reviews and meta-analyses using standard pairwise meta-analytical approaches do not allow comparison of each active intervention against others, making it impossible to evaluate the comparative efficacy of existing psychosocial interventions.

- This leads to uncertainty on which intervention should be considered first choice.

What are the new findings?

- This is the first a network meta-analysis that compared and ranked psychosocial interventions for the treatment of PTSD in adult asylum seekers and refugees.

\section{What do the new findings imply?}

- Cognitive behaviouraltherapy with a trauma-focused component and eye movement desensitisation and reprocessing should be made routinely available to adult asylum seekers and refugees with PTSD resettled in countries irrespective of income category.

- Current evidence should inform the development of evidence-based guidelines and implementation packages.

and conventions exist to protect the rights of migrants and refugees, including their right to mental health, which is fundamental to contribute to the social and economic development of their communities of origin and destination, and to integrate in the host country on a personal, social, and economic level. ${ }^{1}$

Refugees are a subset of forcibly displaced individuals, as the term refugee is a legal definition related to the 1951 United Nations Convention on the rights of refugees. ${ }^{2}$ Thus, not all forcibly displaced individuals are 
recognised as refugees, and many may be asylum seekers or internally displaced people. ${ }^{34}$

According to the United Nations High Commissioner for Refugees (UNHCR), ${ }^{5}$ there are currently more than 30 million refugees and asylum seekers resettled in high-income countries (HICs) and in low/middleincome countries (LMICs). Existing evidence highlights that the experience of forced migration can make this population particularly vulnerable to life stressors with a negative impact on their mental health. ${ }^{6}$ Life stressors may be experienced before, during and after migration, and include mass violence, discrimination, unmet basic needs, uncertainty about the future, concerns for the safety of family members and long-drawn asylum procedures. $^{7-9}$ Consequently, the number of refugees and asylum seekers suffering from mental disorders, in particular post-traumatic stress disorder (PTSD), depression and anxiety, is significantly higher than in populations without a recent history of mass violence. ${ }^{10-12}$

Of these conditions, PTSD is 10 times more frequent in refugees and asylum seekers than in the general population, and represents a major global health problem. ${ }^{10} 13$ Research suggests that psychosocial interventions are effective in treating PTSD and related symptoms in individuals who were exposed to traumatic events. ${ }^{14}{ }^{15}$ However, addressing mental health of refugees represents a challenge for receiving societies. ${ }^{16}$ For many professionals, working with refugees and asylum seekers is associated with additional challenges related to cultural, language and legal barriers. ${ }^{17} 18$ Hence, a number of randomised trials have investigated the efficacy of psychosocial interventions specifically adapted to meet the needs of adult refugees and asylum seekers with PTSD. Systematic reviews and meta-analyses of these studies generally found evidence for the benefits of cognitive behavioural therapy (CBT) with a traumafocused component, eye movement desensitisation and reprocessing (EMDR) and narrative exposure therapy (NET). ${ }^{19-23}$

A major shortcoming of existing systematic reviews and meta-analyses is the use of standard pairwise metaanalytical approaches that does not allow comparison of each active intervention against others, making it impossible to evaluate the comparative efficacy of existing psychosocial interventions. This leads to uncertainty on which intervention should be considered first choice. In refugees with PTSD, providing the most appropriate psychosocial interventions is a priority that could reduce use of pharmacological strategies and hospitalisation. ${ }^{24}$ Against this background, the present systematic review applied network meta-analysis (NMA) techniques to compare psychosocial interventions for the treatment of PTSD in adult asylum seekers and refugees, and to estimate the ranking probabilities of being at each possible rank for each intervention. Ranking treatments in a hierarchical order is a straightforward and user-friendly way to inform practitioners, policy makers, clients, and other stakeholders. ${ }^{25}$

\section{METHODS}

The protocol for this review was registered in the International Prospective Register of Systematic Reviews (PROSPERO), registration number: CRD42019126604.

\section{Selection of studies}

The following bibliographical databases were searched up to 21 February 2020: Cochrane Central Register of randomised trials (CENTRAL), MEDLINE, PTSDpubs PsycINFO, PubMed, CINAHL, EMBASE, Web of Science, WHO's International Clinical Trials Registry Platform and ClinicalTrials.gov. The reference lists of previously published reviews and meta-analyses, and original research articles were additionally scrutinised to identify publications not covered by the original database searches. We applied no publication or language restrictions. Details of the search strategy and screening process are reported in online supplemental material. The selection process was recorded in agreement with the Preferred Reporting Items for Systematic Reviews and Meta-Analyses guidelines specific for NMA, and it was performed by two independent authors (GT, CR) ${ }^{26}$

Studies meeting the following criteria were included: (a) randomised controlled trials (RCTs); (b) including adult participants having an asylum seeker and/or a refugee status, as defined by UNHCR; (c) assessing the efficacy of a psychosocial intervention; (d) comparing psychosocial interventions with inactive interventions like treatment as usual (TAU, defined as any intervention that reflects the usual care in a given treatment setting), waiting list (WL) or any other active psychosocial interventions; (e) having at least $80 \%$ of study participants with a PTSD diagnosis according to the Diagnostic and Statistical Manual of Mental Disorders (DSM), or the International Classification of Diseases (ICD), or with a probable diagnosis according to clinician-led structured interviews, or self-report measures validated for PTSD assessment. ${ }^{2}$ Psychosocial interventions were defined as any psychological and/or social or rehabilitation effort aimed at improving PTSD symptoms, without the use of psychopharmacological agents, ${ }^{27-29}$ that is, the term as it is commonly applied in biomedical literature-as opposed to its use by humanitarian practitioners. Studies in which concomitant use of medications was allowed were not excluded.

\section{Outcome measures}

PTSD symptoms measured on continuous rating scales or structured interviews at postintervention were the primary outcome. Data were extracted from the ClinicianAdministered PTSD Scale (CAPS) ${ }^{30}$ or, if this scale was not available, from the Harvard Trauma Questionnaire (HTQ) ${ }^{31}$ or from any other PTSD rating scale, based on DSM or ICD criteria. Secondary outcomes included depressive symptoms, anxiety symptoms, global functioning, well-being or quality of life, measured with the relative rating scales at postintervention, as well as treatment acceptability, measured as the number of participants who dropped out by any cause at study end-point. 


\section{Data extraction and quality assessment}

Two review authors (GT, CR) independently assessed titles, abstracts and full-texts of potentially relevant articles, and extracted data following the recommendations of the Cochrane Handbook for Systematic Reviews of Interventions. ${ }^{32}$ Two review authors (GT, CR) assessed the methodological quality of included studies using the Cochrane Risk of Bias (RoB) tool. ${ }^{32}$ Overall, studies were classified as having a low risk of bias if three or more domains of the Cochrane RoB were at low risk; studies were classified having a high risk of bias if two or more domains were at high risk; all other cases were assumed to be unclear. Disagreements were resolved by discussion and consensus with a third review author (CB). Details on the quality assessment process and RoB tables are provided in online supplemental material.

For continuous outcomes, we extracted the mean scores and SD at postintervention or, if it was neither available nor inferable from the information available, the mean change from baseline, the SD of these values and the number of participants included in these analyses. For dichotomous outcomes, we extracted the number of participants undergoing the randomisation procedure, and the number of participants leaving the study early for any reason.

\section{Data synthesis}

We performed a standard pair-wise meta-analysis for every comparison and, for each outcome, an NMA in a frequentist framework. The Stata mometa package was used to perform the analyses (Stata/SE 16.1). ${ }^{33}$ This allowed us to include multi-arm trials in the analysis by considering the correlation between the effect sizes of each of their pairwise comparisons. ${ }^{34}$ For continuous outcomes, we pooled the standardised mean differences (SMDs) between treatment arms at postintervention as the included studies measured the outcomes using different rating scales. For dichotomous outcomes, we calculated and pooled ORs with $95 \%$ CIs. Intervention groups that met criteria for the same intervention classification were combined together into a single node following standard approaches. ${ }^{35} 36$ Moreover, studies that compared two or more formats of similar psychosocial interventions with an inactive treatment were included in meta-analysis by combining the respective group arms into a single group. ${ }^{32}$

For any outcome we estimated the ranking probabilities for all treatments of being at each possible rank for each intervention. We obtained a treatment hierarchy using the surface under the cumulative ranking curve (SUCRA) and mean ranks. SUCRA was expressed as a percentage and is interpreted as the percentage of efficacy or safety a treatment achieves in relation to a treatment that would be ranked first without uncertainty. ${ }^{25}$

We asked trial authors to supply data when there was missing or unclear information. When SDs were not reported and not supplied by authors on request, we calculated them based on other measures reported in the study, for example, SEs, t-statistics or $\mathrm{p}$ values, according to Altman and Bland. ${ }^{37}$

For pairwise meta-analyses, we assessed heterogeneity using the $\mathrm{I}^{2}$ statistics, following the interpretation suggested by the Cochrane handbook: $0 \%-40 \%$ : might not be important; 30\%-60\%: may represent moderate heterogeneity; 50\%-90\%: may represent substantial heterogeneity; $75 \%-100 \%$ : considerable heterogeneity. ${ }^{32}$ For the NMA, common heterogeneity across all comparisons was assumed and estimated in each network. ${ }^{38}$

As the assumption of transitivity requires effect modifiers to be equally distributed across the comparisons, we evaluated this assumption by extracting key study characteristics judged to be potential effect modifiers, namely: number of participants included; income level (HIC or LMIC); number of sessions of the intervention. We compared their distribution across interventions in the network, using the Kruskal-Wallis test for continuous variables, and the Fisher's exact test for binary variables. Meta-regressions were additionally performed to evaluate their association with the primary outcome results. For effect modifiers showing evidence that the transitivity assumption might not be met, subgroup analyses were conducted.

Coherence (also known as consistency in NMAs) in a network of treatments refers to the agreement between direct and indirect evidence on the same comparisons. We first checked for any erroneous data abstraction. We then evaluated the presence of incoherence by comparing direct and indirect evidence within each closed loop of nodes, ${ }^{39} 40$ and comparing the goodness of fit for an NMA model that assumes consistency with a model that allows for incoherence in a 'design by treatment interaction model' framework, ${ }^{41-43}$ using the Stata commands mvmeta ${ }^{44}$ and ifplot $t^{45}$ in the Stata network suite. ${ }^{46}$ We also investigated possible incoherence further using a side-splitting approach between comparisons (ie, splitting the total evidence between its direct and indirect component, and comparing them). ${ }^{46}$

We performed a likelihood-ratio test to the consistency model. In case of statistical significance, we implemented random-effects pairwise and network meta-analyses, otherwise we implemented fixed-effects pairwise and network meta-analyses.

Publication bias was assessed for each pairwise comparison only if at least 10 studies provided data for the primary outcome. ${ }^{47} \mathrm{~A}$ global funnel plot analysis was also conducted by evaluating, for the primary outcome, active vs inactive interventions and head-to-head comparisons. We visually inspected the funnel plot, and conducted a test for asymmetry with the Egger's regression test. ${ }^{48}$ For fixed-effects model, we produced contour enhanced funnel plots to help distinguish publication bias from other types of asymmetry. ${ }^{49}$

For the primary outcome, certainty of evidence was assessed through the Confidence in Network MetaAnalysis application (http://cinema.ispm.ch/), an adaptation of the Grading of Recommendations Assessment, Development and Evaluation (GRADE) approach for NMAs. ${ }^{50} 51$

A priori subgroup analyses by country income (HICs vs LMICs) and level of intervention (individual vs group intervention) were conducted. Sensitivity analyses excluding trials with high risk of bias and excluding 


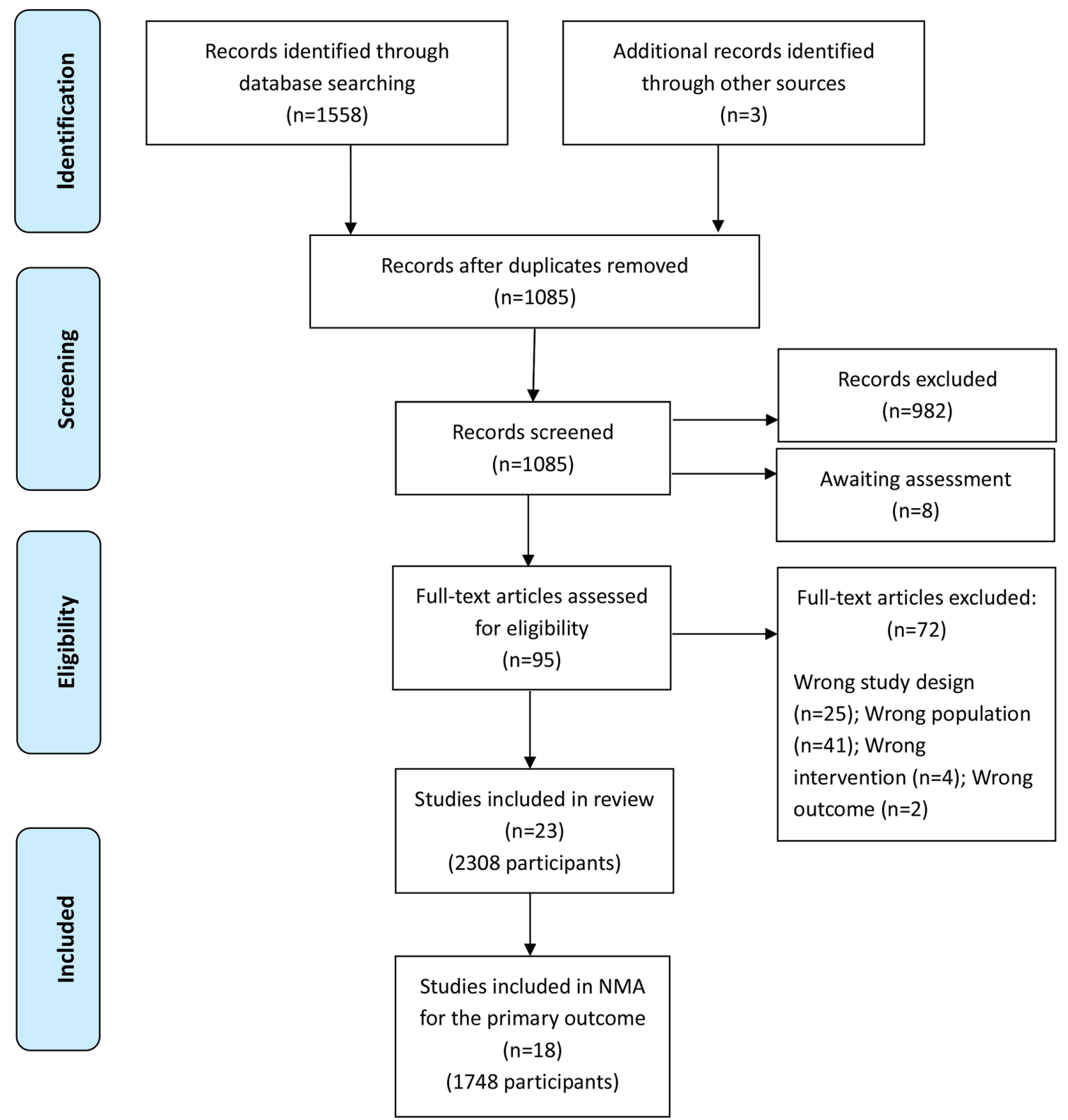

Figure 1 Preferred Reporting Items for Systematic Reviews and Meta-Analyses flow-chart diagram. NMA, network metaanalysis.

studies without a formal PTSD diagnosis were carried out.

\section{Patient and public involvement}

Patient and public involvement was not conducted as part of this review.

\section{RESULTS}

\section{Characteristics of included studies}

The electronic search yielded a total number of 1085 records (after removal of duplicates). After title and abstract screening, 95 full text papers were considered for inclusion, of which 23 studies, including 2308 participants, fulfilled the eligibility criteria and were included in the systematic review ${ }^{52-74}$ (figure 1). References of excluded studies and reasons for exclusion are reported in online supplemental material.

Eleven studies employed a WL as a control condition, nine compared a psychosocial intervention with TAU or no treatment and seven studies compared psychosocial interventions head-to-head. In 11 studies, participants received concomitant psychopharmacological medication. The study sample sizes ranged from 10 to 694. Sixteen studies were conducted in HICs, and seven in LMICs. Eleven studies recruited participants that were homogeneous in terms of nationality, three did not specify the participants' country of origin, while in the remaining studies a range of different nationalities were included. Nineteen studies recruited participants with a formal PTSD diagnosis using the following diagnostic tools: MINI International Neuropsychiatric 
Interview, the Composite International Diagnostic Interview, the Structured Clinical Interview for DSM-IV, the CAPS, the Post-Traumatic Stress Diagnostic scale, the ICD-10 Classification of Mental and Behavioural Disorders (ICD) and the DSM; and four studies recruited participants with a probable PTSD diagnosis using validated self-report instruments including the HTQ, the PTSD Checklist-Civilian six-item version and the Impact of Event Scale-Revised scales (online supplemental material).

Thirteen studies were conducted in healthcare settings, six in community settings in refugee camps, two in social-care settings, one in a place of worship and one in the community. The mean age of included participants was 36.2 years (range from included studies: 30.9-51.8). Three studies included only women, while in the remaining studies the mean proportion of included women was $64 \%$ (range: $15 \%-77 \%$ ) (online supplemental material).

The following interventions were included: coffee and family education and support (one study); CBT and related adapted protocols with a trauma component (eight studies); cognitive restructuring (one study); EMDR and related protocols (five studies); exposure therapy (one study); NET (seven studies); self-help plus $(\mathrm{SH}+)$ (one study); stress inoculation training (one study); stabilisation therapy (two studies); stress management (one study); supportive counselling (one studies); trauma counselling (one study). Psychosocial interventions belonging to the same theoretical model were brought together into a single node (online supplemental material).

Five of 23 studies were at high risk of bias on two or more items of the Cochrane RoB tool, fifteen were at low risk of bias, and three were unclear (online supplemental material).

\section{PTSD symptoms and overall acceptability}

Eighteen studies contributed to the analysis on PTSD symptoms at postintervention, while five did not have data suitable for reanalysis. ${ }^{5463646773}$ Figure 2 shows the network plot of interventions for this outcome, while table 1 reports the results of the NMA. Both CBT (SMD $=-1.41 ; 95 \%$ CI -2.43 to -0.38$)$ and EMDR $(\mathrm{SMD}=-1.30 ; 95 \% \mathrm{CI}-2.40$ to -0.20$)$ were significantly more effective than WL (figure 3).

CBT was also associated with higher decrease in PTSD symptoms as compared with TAU (SMD $-1.51 ; 95 \%$ CI -2.67 to -0.36 ) (table 1 ). For the other interventions, the difference with $\mathrm{WL}$ and TAU was not significant, and no differences between active interventions emerged. Generally, no relevant heterogeneity emerged for pairwise comparisons, except for two comparisons: EMDR versus $W L\left(I^{2}=79.4\right)$ and $C B T$ versus $W L\left(I^{2}=93.3\right)$. Intraloop incoherence emerged for one loop, involving TAU, NET, SSM and CBT. The test for overall network heterogeneity was significant (estimated between-study SD 1.34, $\mathrm{p}$ value $<0.001$ in the inconsistency model; SD 1.04, $\mathrm{p}$ value $<0.001$ in the consistency model), while the designby-treatment test did not reveal incoherence ( $p$ value 0.97). Results of the NMA were consistent with results from pairwise meta-analysis, except for the comparisons $\mathrm{SH}+$ versus TAU (significant in the direct estimate only) and CBT versus WL (significant in the mixed estimate only). By splitting direct and indirect evidence for each comparison, we found no evidence for disagreement between these two pieces of evidence for any of the comparisons. We found no clear evidence of violations of the transitivity assumption when comparing characteristics of studies across interventions, with the exception of income level, which was not equally distributed across interventions (online supplemental material). Global funnel plot analysis for the primary outcome showed no

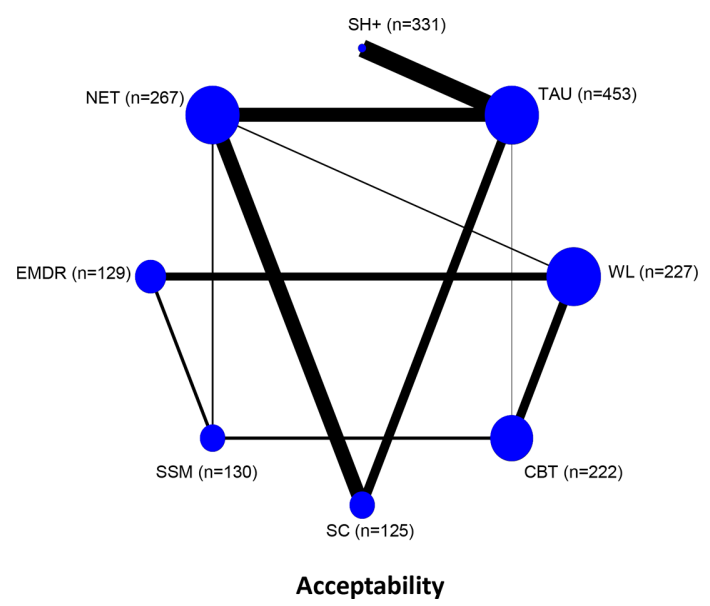

Figure 2 Network plot of evidence for PTSD symptoms and acceptability. The thickness of edges is proportional to the precision of each direct estimate (inverse of the variance), and the size of nodes is proportional to the number of studies including that intervention. CBT, cognitive behavioural therapy; EMDR, eye movement desensitisation and reprocessing; $\mathrm{n}$, number of participants allocated to intervention; NET, narrative exposure therapy; PTSD, post-traumaticstress disorder; SC, supportive/trauma counselling; SH+, self-help plus; SSM, stabilisation/stress management; TAU, treatment as usual; WL, waitlist. 

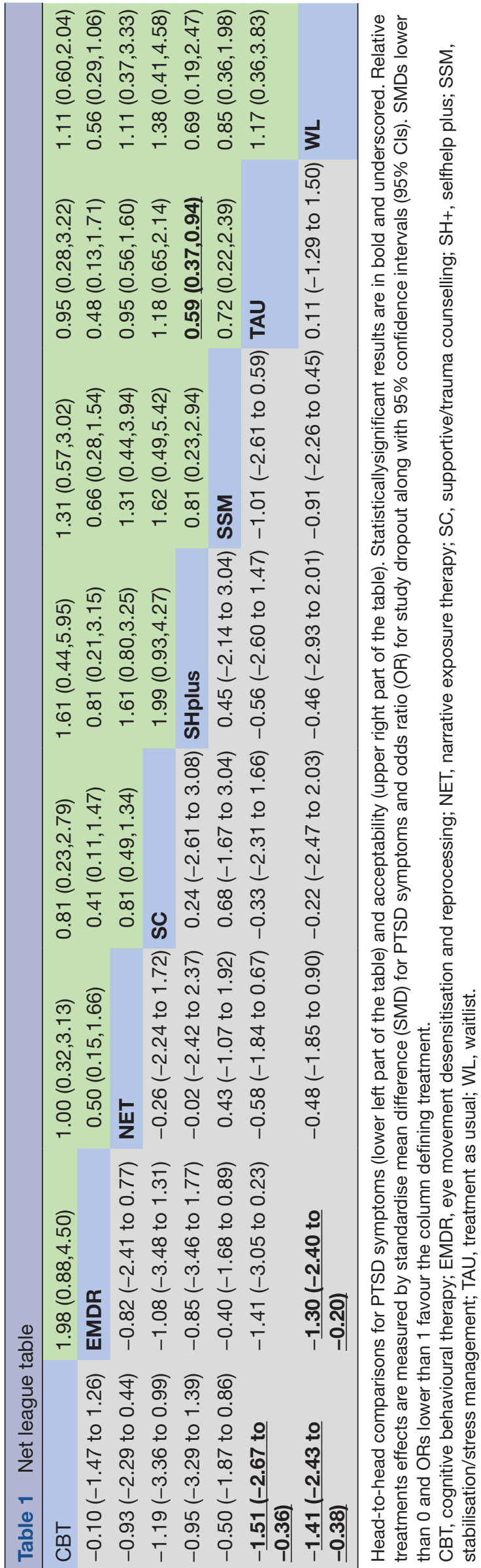

evidence of publication bias $(\mathrm{p}=0.178)$ (online supplemental material).

CBT and EMDR ranked best according to the mean SUCRA. Compared with TAU, the certainty of evidence was 'moderate' for CBT, while it was 'low' for the comparisons $\mathrm{CBT}$ and EMDR versus $\mathrm{WL}$, due to high heterogeneity. For most of the other comparisons the certainty of evidence was "low' or 'very low' mainly due to imprecision of results and heterogeneity (online supplemental material).

Regarding acceptability, no intervention had less dropouts than inactive interventions, and no differences between active interventions emerged (table 1). The network did not show significant overall incoherence (design-by-treatment test, $\mathrm{p}$ value 0.954 ) nor heterogeneity (estimated between-studies SD 0.50, $\mathrm{p}$ value 0.201 in the inconsistency model; SD 0.13 , p value 0.462 in the consistency model). No intra-loop incoherence emerged, and results of the NMA were consistent with results from pairwise meta-analyses (online supplemental material).

Results of sensitivity analyses generally confirmed a better performance of CBT and EMDR in comparison with inactive intervention, and their best ranking according to the mean SUCRA. The overall heterogeneity remained significant in both sensitivity analyses. The intra-loop incoherence of the loop TAU-NETSSM-CBT became marginally statistically non-significant when studies with high risk of bias were excluded, while it emerged for another loop involving TAU, NET, SSM and SC, when studies without a formal PTSD diagnosis were excluded. Nevertheless, no overall incoherence emerged, and statistical agreement between direct and indirect estimates was confirmed (online supplemental material).

Subgroup analysis by country income level revealed that most studies with data on PTSD outcome were conducted in HICs (12 studies), with only 6 studies conducted in LMICs. For LMICs, incoherence could not be assessed due to unavailability of pairs of treatments with both direct and indirect comparisons. CBT, NET and SSM were significantly more effective than TAU in HICs, and CBT and EMDR were associated with higher decrease in PTSD symptoms than WL in LMICs, with a better performance of CBT. Substantial heterogeneity was detected for some pairwise comparisons involving CBT and EMDR versus WL, but the overall heterogeneity, although significant, decreased and, for studies in HICs, no overall incoherence and intra-loop incoherence emerged. Statistical agreement between direct and indirect estimates was found for all comparisons. Subgroup analysis by level of intervention revealed that in most studies with the primary outcome available, the interventions were delivered individually (14 studies). In this subgroup, heterogeneity was found in the only comparison (CBT vs WL) with three studies available, and a significant overall incoherence emerged. However, results from pairwise comparisons were consistent with those from the primary analysis. In terms of group interventions, tests for heterogeneity and 


\section{Efficacy: PTSD symptoms}

\section{Reference treatment: Waitlist}

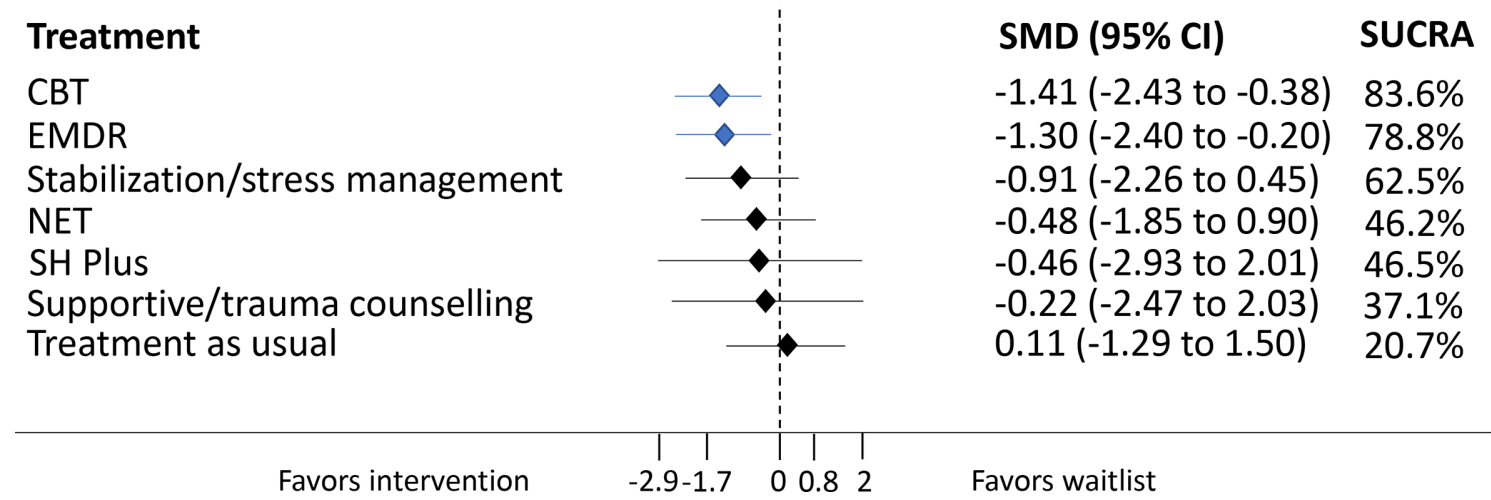

Figure 3 Forest plots comparing each treatment with waitlist for PTSD symptoms with the corresponding ranking probability (SUCRA) for each intervention. Statistically significant results are coloured in blue. CBT, cognitive behavioural therapy; $\mathrm{Cl}$, confidence interval; EMDR, eye movement desensitisation and reprocessing; NET, narrative exposure therapy; PTSD, posttraumaticstress disorder; $\mathrm{SH}+$, self-help plus; SMD, standardised mean difference; SUCRA, surface under the cumulative ranking.

incoherence were not possible due to the limited available data (four studies) (online supplemental material).

\section{Secondary outcomes}

Results for depression and anxiety are reported in online supplemental material, with 13 studies contributing to the analysis on depressive symptoms and 11 studies on anxiety symptoms. CBT was associated with a higher reduction of depressive symptoms compared with WL, and with higher reduction of anxiety symptoms compared with TAU and $\mathrm{WL}$. No significant differences emerged between active interventions. A significant overall heterogeneity ( $p$ value $<0.001$ ) emerged for both depression (SD 1.88, in the inconsistency model; SD 1.65, in the consistency model) and anxiety (SD 1.86, in the inconsistency model; SD 1.70, in the consistency model) and substantial heterogeneity was detected for some pairwise comparisons involving mostly CBT and WL ( $\mathrm{I}^{2} 95.2 \%$ and $88.0 \%$ for depression; $92.2 \%$ and $86.0 \%$ for anxiety). However, for these outcomes no overall incoherence (design-bytreatment test, depression $\mathrm{p}$ value 0.631 and anxiety $\mathrm{p}$ value 0.694 ) and intra-loop incoherence emerged, and generally, results of the NMA were consistent with results from pairwise meta-analyses. There was statistical agreement between direct and indirect estimates (online supplemental material).

For the analyses on functioning, quality of life and well-being, the interpretation is limited due to restricted number of studies available. For functioning, only one indirect comparison between SSM versus WL was computed, which did not reveal any significant difference. For quality of life and well-being, results from the net league table showed that $\mathrm{SH}+$ was significantly more effective than TAU (SMD 0.41; 95\% CI 0.25 to 0.57), consistently with the results from pairwise meta-analysis.
Tests for heterogeneity and incoherence were not possible due to the limited available data (online supplemental material).

\section{DISCUSSION}

The present NMA estimated the relative treatment effects of various psychosocial interventions for asylum seekers and refugees with PTSD in both HICs and LMICs. Notably, the probability of the ranking order for each intervention was calculated, allowing to rank interventions even when direct head-to-head studies were not available.

A total of 23 studies and 2308 participants were included in this review. CBT, NET and EMDR were the most represented interventions in terms of included studies. CBT and EMDR appeared to have the greatest effects in reducing PTSD symptoms, with the highest probability of being at the top of the hierarchy, as shown by the cumulative probability plots. No significant evidence of efficacy for other interventions was found, probably due to the limited number of the included studies. Results of sensitivity analyses generally confirmed a better performance for CBT and EMDR, while interpretation of subgroup analyses was heavily limited by the low number of studies available in each subgroup. However, results were consistent with the primary analyses.

Previous meta-analyses were able to show the benefit of trauma-focused interventions for refugees and asylum seekers with PTSD, and suggested a promising role for EMDR and NET. ${ }^{19-23}$ However, the comparative and relative efficacy of these interventions could not be ascertained. In the present review, we showed that, among all interventions, those based on CBT with a trauma-focused component were the most effective, followed by EMDR. 
In contrast with Kip and colleagues, ${ }^{19}$ who highlighted that NET was effective at follow-up assessments, we failed to show a significant effect of NET for PTSD symptoms at postintervention. However, NET appeared more effective than TAU in the subgroup of studies conducted in HICs, consistently with Nosè and colleagues. ${ }^{20}$ Given that only two studies on the efficacy of NET were conducted in LMICs, we argue that current evidence base on NET in low resources settings needs to be expanded before drawing firm conclusions on its overall beneficial effects.

The finding that CBT and EMDR are effective treatments for PTSD is in line with the literature on the efficacy of psychosocial interventions for PTSD in general. ${ }^{14} 15$ However, the current findings should be interpreted bearing in mind some limitations. First, the overall number of included studies was relatively low, with a limited total number of participants contributing to the primary analysis, and with relatively few direct comparisons between active interventions. Therefore, most of our evidence was based on indirect treatment comparisons, which are more susceptible to bias. In addition, some secondary outcomes like functioning, wellbeing and quality of life, which play a relevant role in this particular population, were poorly reported by the original studies, leading to poorly populated and connected networks. Second, we included interventions which did not target PTSD as primary outcome, leading to potential differences on effect. Moreover, the included studies were heterogeneous in terms of a number of clinical and methodological aspects, such as participants' background and country of resettlement, time since resettlement, outcome measures, diagnostic criteria, treatment content and modalities of delivering the interventions. These aspects likely contributed to the high level of statistical heterogeneity that was detected, and that was not fully explained by subgroup and sensitivity analyses. Heterogeneity, together with imprecision of CIs around the treatment estimates, was responsible for an overall judgement of low confidence according to the GRADE approach. We note, however, that the overall coherence, which is a key aspect to consider in NMA, ${ }^{75}$ appeared to be well preserved for most analyses. Third, we made the a priori choice of analysing data at postintervention only, as we anticipated that in a relevant number of studies long term data were lacking and networks could have been poorly connected, leaving uncertainty on the longterm effect of psychosocial interventions. Moreover, 11 studies included participants that received concomitant psychopharmacological medications. Although dosages remained stable throughout the intervention period, and no differences between treatment groups were found with respect to medication exposure, we cannot exclude that having received pharmacological treatment could have represented a source of variability, with a potential impact on study outcomes. Fourth, as no comparison included more than 10 studies, visual inspection of funnel plots for single comparisons was not conducted, leaving a potential risk of publication bias. However, a global funnel plot analysis for the primary outcome showed no evidence of publication bias. Finally, we found no clear evidence of violations of the transitivity assumption when comparing characteristics of studies across interventions. However, in some outcomes, the number of studies per comparison was small and the case of intransitivity cannot be completely excluded.

All these limitations should be considered within the scope of the challenging context within which many studies have been undertaken and the complex needs of the target populations. ${ }^{76}$ Personal narratives, vulnerabilities, barriers to access health services, cultural perspectives on mental illness and help-seeking, and variation in cultural concepts of distress have been recognised as key variables influencing efficacy of interventions with refugees and asylum seekers. ${ }^{7}$ Furthermore, many mental health professionals may have reservations to work with refugees as this work is often perceived as more challenging due to anticipated language, cultural and legal difficulties. ${ }^{18}$ This, in turn, may influence treatment efficacy. In addition, refugees and asylum seekers may be less willing to engage in psychosocial intervention studies because of the stigma associated with psychological problems and the lack of knowledge about reasons for being offered a psychosocial intervention. ${ }^{77-79}$ Language is another obstacle for both participants and investigators, and instrument translation and involvement of native speakers for conducting the assessments is generally required. ${ }^{80}$ Moreover, tools used for identifying groups who need mental health attention may not be adequate for different cultural groups, with a potential negative influence in trust on study outcomes.

A number of implications for research, policy, and practice can be drawn from our findings. Larger and higher quality studies with long-term assessments of intervention efficacy are needed to consolidate findings and to enhance our understanding of the sustainability of the effects of psychosocial interventions. Further direct comparisons between active interventions are needed to determine comparative efficacy in a more accurate way, and the present NMA would suggest that CBT with a trauma-focused component may be employed as effective reference standard. Moreover, research is needed to determine whether effective interventions for regular PTSD are also effective for the ICD11 complex PTSD diagnosis, as a subset of refugees might meet criteria for that specific diagnosis. ${ }^{81}$ Future studies should further examine common therapeutic factors that are purportedly beneficial, and that could be considered to adapt the interventions based on specific needs of the refugee population. It has been argued that evidence-based treatments that are culturally adapted may be more effective for members of the cultural group for which the treatment was adapted. ${ }^{82}$ This adaptation process may facilitate engagement of refugees and asylum seekers with services, and may optimise intervention acceptability and appropriateness. ${ }^{83}$ Engagement of refugees with services may additionally be facilitated by improving interpreter 
and facilitator services which can, in turn, improve the implementation and outcome of psychosocial interventions. ${ }^{84}$ Based on these considerations, a new generation of studies have recently been designed to demonstrate the effectiveness of culturally adapted psychosocial interventions delivered by non-specialist trained facilitators, or lay counsellors, sharing the same cultural background of the target refugee population. ${ }^{85-89}$ An added value of employing trained non-specialist facilitators includes lower healthcare costs, which may be particularly relevant in low-resource settings. ${ }^{90} 91$ Finally, future studies should additionally investigate the efficacy, feasibility and sustainability of psychosocial interventions delivered through the use of synchronous and asynchronous online communication devices. ${ }^{849293}$ Evidence on virtual delivery of psychotherapies highlighted that they can be as effective as in-person delivery, but with the added value of reducing stigma, costs, and also interpersonal contacts that, in reference to the current COVID-19 pandemic, are potentially risky. ${ }^{94}$ This aspect may be particularly important for asylum seekers and refugees who may be subject to isolation measures often after traumatic separations, physical distancing with complete loss of social networks, and restriction of movement for relatively long periods. $^{96}$

\section{CONCLUSION}

Altogether, the current findings suggest that CBT with a trauma-focused component and EMDR are effective in treating PTSD. More research is needed with regard to other treatment forms. Accordingly, and given the pressing mental health needs of asylum seekers and refugees, these psychosocial interventions should be made routinely available to adult asylum seekers and refugees with PTSD resettled in countries irrespective of income category. Current and future evidence should inform the development of evidence-based guidelines and implementation packages, ${ }^{76}$ aiming to guarantee that all people have equitable access to high-quality mental healthcare.

\footnotetext{
Author affiliations

${ }^{1}$ Cochrane Global Mental Health and WHO Collaborating Centre for Research and Training in Mental Health and Service Evaluation, Department of Neuroscience, Biomedicine and Movement Sciences, Section of Psychiatry, University of Verona, Verona, Italy

${ }^{2}$ Department of Clinical, Neuro, and Developmental Psychology, Amsterdam Public Health Institute, and WHO Collaborating Centre for Research and Dissemination of Psychological Interventions, Vrije Universiteit Amsterdam, Amsterdam, Netherlands ${ }^{3}$ Institute of Primary Health Care (BIHAM), University of Bern, Bern, Switzerland ${ }^{4}$ Institute of Psychology, University of Münster, Munster, Germany

${ }^{5}$ Section of Global Health, Department of Public Health, University of Copenhagen, Kobenhavn, Denmark

${ }^{6}$ Department of Mental Health, Bloomberg School of Public Health, Johns Hopkins University, Baltimore, Maryland, USA

${ }^{7}$ Peter C. Alderman Program for Global Mental Health, HealthRight International, New York, New York, USA

Acknowledgements With thanks Dr Asena Yurtsever for providing information.

Contributors GT and CB conceptualised the network meta-analysis and wrote the protocol. GT and CR screened titles and abstracts for inclusion and inspected the full texts for inclusion. GT and CR performed data extraction and quality
}

assessment. GT, MP and MN took part in collecting data. Analysis was performed by FT, CDG and GO. GT wrote the first manuscript draft. CB and MP reviewed it. Successive versions have been written with feedback from PC, AK, NM, MS, WT. All authors read and approved the final manuscript.

Funding This research received no specific grant from any funding agency, commercial or not-for-profit sectors

Competing interests None declared.

Patient consent for publication Not required.

Provenance and peer review Not commissioned; externally peer reviewed.

Data availability statement All data relevant to the study are included in the article or uploaded as supplementary information. All data relevant to the study are included in the article or uploaded as supplementary information.

Supplemental material This content has been supplied by the author(s). It has not been vetted by BMJ Publishing Group Limited (BMJ) and may not have been peer-reviewed. Any opinions or recommendations discussed are solely those of the author(s) and are not endorsed by BMJ. BMJ disclaims all liability and responsibility arising from any reliance placed on the content. Where the content includes any translated material, BMJ does not warrant the accuracy and reliability of the translations (including but not limited to local regulations, clinical guidelines, terminology, drug names and drug dosages), and is not responsible for any error and/or omissions arising from translation and adaptation or otherwise.

Open access This is an open access article distributed in accordance with the Creative Commons Attribution Non Commercial (CC BY-NC 4.0) license, which permits others to distribute, remix, adapt, build upon this work non-commercially, and license their derivative works on different terms, provided the original work is properly cited, appropriate credit is given, any changes made indicated, and the use is non-commercial. See: http://creativecommons.org/licenses/by-nc/4.0/.

\section{REFERENCES}

1 Abubakar I, Aldridge RW, Devakumar D, et al. The UCL-Lancet Commission on migration and health: the health of a world on the move. Lancet 2018;392:2606-54.

2 General Assembly UN. UNHCR - Protocol Relating to the Status of Refugees, 1951. Available: http://www.unhcr.org/3b66c2aa10.html [Accessed 8 Jun 2020].

3 IOM International Organization for Migration. Glossary on migration, International migration law series No. 25, 2011. Available: https:// publications.iom.int/system/files/pdf/iml25_1.pdf [Accessed 8 Jun 2020].

4 IOM International Organization for Migration. World migration report 2020, 2020. Available: https://publications.iom.int/system/files/pdf/ wmr_2020.pdf [Accessed 8 Jun 2020].

5 UNHCR. Global trends: forced displacement in 2019, 2020. Available: https://www.unhcr.org/5ee200e37.pdf [Accessed 8 Jun 2020].

6 Lund C, Brooke-Sumner C, Baingana F, et al. Social determinants of mental disorders and the sustainable development goals: a systematic review of reviews. Lancet Psychiatry 2018;5:357-69.

7 Chaplin L, Ng L, Katona C. Refugee mental health research: challenges and policy implications. BJPsych Open 2020;6:e102.

8 Giacco D. Identifying the critical time points for mental health of asylum seekers and refugees in high-income countries. Epidemiol Psychiatr Sci 2019;29:e61.

9 Henkelmann J-R, de Best S, Deckers C, et al. Anxiety, depression and post-traumatic stress disorder in refugees resettling in highincome countries: systematic review and meta-analysis. BJPsych Open 2020;6:e68.

10 Blackmore R, Boyle JA, Fazel M, et al. The prevalence of mental illness in refugees and asylum seekers: a systematic review and meta-analysis. PLoS Med 2020;17:e1003337.

11 Priebe S, Giacco D, El-Nagib R. Public health aspects of mental health among migrants and refugees: a review of the evidence on mental health care for refugees, asylum seekers and irregular migrants in the who European region. Copenhagen: WHO Regional Office for Europe, 2016.

12 Turrini G, Purgato M, Ballette F, et al. Common mental disorders in asylum seekers and refugees: umbrella review of prevalence and intervention studies. Int J Ment Health Syst 2017;11:51.

13 Fazel M, Wheeler J, Danesh J. Prevalence of serious mental disorder in 7000 refugees resettled in Western countries: a systematic review. Lancet 2005;365:1309-14. 
14 Cusack K, Jonas DE, Forneris CA, et al. Psychological treatments for adults with posttraumatic stress disorder: a systematic review and meta-analysis. Clin Psychol Rev 2016;43:128-41.

15 Hoppen TH, Morina N. Is high-quality of trials associated with lower treatment efficacy? A meta-analysis on the association between study quality and effect sizes of psychological interventions for pediatric PTSD. Clin Psychol Rev 2020;78:101855.

16 Echterhoff G, Hellmann JH, Back MD, et al. Psychological antecedents of refugee integration (PARI). Perspectives on Psychological Science 2020;15:856-79.

17 Salami B, Salma J, Hegadoren K. Access and utilization of mental health services for immigrants and refugees: perspectives of immigrant service providers. Int $J$ Ment Health Nurs 2019;28:152-61.

18 Schlechter P, Hellmann JH, Wingbermühle P. Which psychological characteristics influence therapists' readiness to work with refugees? Clin Psychol Psychother 2020.

19 Kip A, Priebe S, Holling H, et al. Psychological interventions for posttraumatic stress disorder and depression in refugees: a metaanalysis of randomized controlled trials. Clin Psychol Psychother 2020;27:489-503.

20 Nosè $\mathrm{M}$, Ballette $\mathrm{F}$, Bighelli I, et al. Psychosocial interventions for post-traumatic stress disorder in refugees and asylum seekers resettled in high-income countries: systematic review and metaanalysis. PLoS One 2017;12:e0171030.

21 Thompson CT, Vidgen A, Roberts NP. Psychological interventions for post-traumatic stress disorder in refugees and asylum seekers: a systematic review and meta-analysis. Clin Psychol Rev 2018;63:66-79.

22 Uphoff E, Robertson L, Cabieses B, et al. An overview of systematic reviews on mental health promotion, prevention, and treatment of common mental disorders for refugees, asylum seekers, and internally displaced persons. Cochrane Database Syst Rev 2020;9:CD013458

23 Turrini G, Purgato M, Acarturk C, et al. Efficacy and acceptability of psychosocial interventions in asylum seekers and refugees: systematic review and meta-analysis. Epidemiol Psychiatr Sci 2019;28:376-88.

24 Murray KE, Davidson GR, Schweitzer RD. Review of refugee mental health interventions following resettlement: best practices and recommendations. Am J Orthopsychiatry 2010;80:576-85.

25 Salanti G, Ades AE, loannidis JPA. Graphical methods and numerical summaries for presenting results from multiple-treatment metaanalysis: an overview and tutorial. J Clin Epidemiol 2011;64:163-71.

26 Hutton B, Salanti G, Caldwell DM, et al. The PRISMA extension statement for reporting of systematic reviews incorporating network meta-analyses of health care interventions: checklist and explanations. Ann Intern Med 2015;162:777-84

27 Inter-Agency Standing Committee (IASC). IASC guidelines on mental health and psychosocial support in emergency settings, 2007. Available: https://www.who.int/mental health/emergencies/ guidelines iasc_mental_health_psychosocial_june_2007.pdf [Accessed 1 Jul 2020].

28 Tol WA, Purgato M, Bass JK, et al. Mental health and psychosocial support in humanitarian settings: a public mental health perspective. Epidemiol Psychiatr Sci 2015;24:484-94.

29 Tol WA, Barbui C, Galappatti A, et al. Mental health and psychosocial support in humanitarian settings: linking practice and research. Lancet 2011;378:1581-91.

30 Blake DD, Weathers FW, Nagy LM, et al. The development of a Clinician-Administered PTSD scale. J Trauma Stress 1995;8:75-90.

31 Mollica RF, Caspi-Yavin Y, Bollini P, et al. The Harvard trauma questionnaire. validating a cross-cultural instrument for measuring torture, trauma, and posttraumatic stress disorder in Indochinese refugees. J Nerv Ment Dis 1992;180:111-6.

32 Higgins JPT, Thomas J, Chandler J, eds. Cochrane. Cochrane Handbook for Systematic Reviews of Interventions version 6.1 (updated September 2020). Cochrane, 2020.

33 White IR. Multivariate Random-effects meta-analysis. Stata $J$ 2009;9:40-56

34 White IR. Multivariate Random-effects meta-regression: updates to Mvmeta. Stata J 2011;11:255-70.

35 Shi C, Westby M, Norman G, et al. Node-making processes in network meta-analysis of nonpharmacological interventions should be well planned and reported. J Clin Epidemiol 2018;101:124-5.

36 Ter Veer E, van Oijen MGH, van Laarhoven HWM. The use of (network) meta-analysis in clinical oncology. Front Oncol 2019;9:822.

37 Altman DG, Bland JM. Detecting skewness from summary information. BMJ 1996;313:1200.

38 Lu G, Ades AE. Combination of direct and indirect evidence in mixed treatment comparisons. Stat Med 2004;23:3105-24.
39 Bucher HC, Guyatt GH, Griffith LE, et al. The results of direct and indirect treatment comparisons in meta-analysis of randomized controlled trials. J Clin Epidemiol 1997;50:683-91.

40 Krahn U, Binder H, König J. A graphical tool for locating inconsistency in network meta-analyses. BMC Med Res Methodol 2013;13:35

41 Higgins JPT, Jackson D, Barrett JK, et al. Consistency and inconsistency in network meta-analysis: concepts and models for multi-arm studies. Res Synth Methods 2012;3:98-110.

42 Jackson D, Barrett JK, Rice S, et al. A design-by-treatment interaction model for network meta-analysis with random inconsistency effects. Stat Med 2014;33:3639-54.

43 Veroniki AA, Vasiliadis HS, Higgins JPT, et al. Evaluation of inconsistency in networks of interventions. Int J Epidemiol 2013:42:332-45.

44 White IR. Network meta-analysis. Stata J 2015;15:951-85

45 Chaimani A, Higgins JPT, Mavridis D, et al. Graphical tools for network meta-analysis in STATA. PLoS One 2013;8:e76654.

46 White IR. Network meta-analysis. Palmer T, Sterne J, eds. MetaAnalysis in Stata: an updated collection from the Stata Journal. 2. College Station, TX: Stata Press, 2016

47 Sterne JAC, Sutton AJ, loannidis JPA, et al. Recommendations for examining and interpreting funnel plot asymmetry in meta-analyses of randomised controlled trials. BMJ 2011;343:d4002.

48 Egger M, Davey Smith G, Schneider M, et al. Bias in meta-analysis detected by a simple, graphical test. BMJ 1997;315:629-34.

49 Peters JL, Sutton AJ, Jones DR, et al. Contour-enhanced metaanalysis funnel plots help distinguish publication bias from other causes of asymmetry. J Clin Epidemiol 2008;61:991-6.

50 Nikolakopoulou A, Higgins JPT, Papakonstantinou T, et al. Cinema: an approach for assessing confidence in the results of a network meta-analysis. PLoS Med 2020;17:e1003082.

51 Salanti G, Del Giovane C, Chaimani A, et al. Evaluating the quality of evidence from a network meta-analysis. PLoS One 2014;9:e99682.

52 Acarturk C, Konuk E, Cetinkaya M, et al. The efficacy of eye movement desensitization and reprocessing for post-traumatic stress disorder and depression among Syrian refugees: results of a randomized controlled trial. Psychol Med 2016;46:2583-93.

53 Acarturk C, Konuk E, Cetinkaya M, et al. EMDR for Syrian refugees with posttraumatic stress disorder symptoms: results of a pilot randomized controlled trial. Eur J Psychotraumatol 2015:6:27414

54 Adenauer H, Catani C, Gola H, et al. Narrative exposure therapy for PTSD increases top-down processing of aversive stimuli--evidence from a randomized controlled treatment trial. BMC Neurosci 2011;12:127.

55 Buhmann CB, Nordentoft M, Ekstroem M, et al. The effect of flexible cognitive-behavioural therapy and medical treatment, including antidepressants on post-traumatic stress disorder and depression in traumatised refugees: pragmatic randomised controlled clinical trial. Br J Psychiatry 2016;208:252-9.

56 Carlsson J, Sonne C, Vindbjerg E, et al. Stress management versus cognitive restructuring in trauma-affected refugees-A pragmatic randomised study. Psychiatry Res 2018;266:116-23.

57 Hensel-Dittmann D, Schauer M, Ruf M, et al. Treatment of traumatized victims of war and torture: a randomized controlled comparison of narrative exposure therapy and stress inoculation training. Psychother Psychosom 2011;80:345-52.

58 Hijazi AM, Lumley MA, Ziadni MS, et al. Brief narrative exposure therapy for posttraumatic stress in Iraqi refugees: a preliminary randomized clinical trial. J Trauma Stress 2014:27:314-22.

59 Hinton DE, Chhean D, Pich V, et al. A randomized controlled trial of cognitive-behavior therapy for Cambodian refugees with treatmentresistant PTSD and panic attacks: a cross-over design. J Trauma Stress 2005;18:617-29.

60 Hinton DE, Hofmann SG, Pollack MH, et al. Mechanisms of efficacy of CBT for Cambodian refugees with PTSD: improvement in emotion regulation and orthostatic blood pressure response. CNS Neurosci Ther 2009;15:255-63.

61 Hinton DE, Pham T, Tran M, et al. Cbt for Vietnamese refugees with treatment-resistant PTSD and panic attacks: a pilot study. J Trauma Stress 2004;17:429-33.

62 Liedl A, Müller J, Morina N, et al. Retracted : Physical Activity within a CBT Intervention Improves Coping with Pain in Traumatized Refugees: Results of a Randomized Controlled Design. Pain Medicine 2011;12:234-45.

63 Neuner F, Kurreck S, Ruf M, et al. Can asylum-seekers with posttraumatic stress disorder be successfully treated? A randomized controlled pilot study. Cogn Behav Ther 2010;39:81-91.

64 Neuner F, Onyut PL, Ertl V, et al. Treatment of posttraumatic stress disorder by trained lay counselors in an African refugee settlement: a randomized controlled trial. J Consult Clin Psychol 2008;76:686-94. 
65 Neuner F, Schauer M, Klaschik C, et al. A comparison of narrative exposure therapy, supportive counseling, and psychoeducation for treating posttraumatic stress disorder in an African refugee settlement. J Consult Clin Psychol 2004;72:579-87.

66 Otto MW, Hinton D, Korbly NB, et al. Treatment of pharmacotherapyrefractory posttraumatic stress disorder among Cambodian refugees: a pilot study of combination treatment with cognitive-behavior therapy vs sertraline alone. Behav Res Ther 2003:41:1271-6.

67 Paunovic N, Ost LG. Cognitive-behavior therapy vs exposure therapy in the treatment of PTSD in refugees. Behav Res Ther 2001;39:1183-97.

68 Shaw SA, Ward KP, Pillai V, et al. A group mental health randomized controlled trial for female refugees in Malaysia. Am J Orthopsychiatry 2019;89:665-74.

69 Stenmark H, Catani C, Neuner F, et al. Treating PTSD in refugees and asylum seekers within the general health care system. A randomized controlled multicenter study. Behav Res Ther 2013;51:641-7.

70 Ter Heide FJJ, Mooren TM, Kleijn W, et al. EMDR versus stabilisation in traumatised asylum seekers and refugees: results of a pilot study. Eur J Psychotraumatol 2011;2. doi:10.3402/ejpt.v2i0.5881. [Epub ahead of print: 1608 2011].

71 Ter Heide FJJ, Mooren TM, van de Schoot R, et al. Eye movement desensitisation and reprocessing therapy $\mathrm{V}$. stabilisation as usual for refugees: randomised controlled trial. $\mathrm{Br} J$ Psychiatry 2016;209:311-8.

72 Tol WA, Leku MR, Lakin DP, et al. Guided self-help to reduce psychological distress in South Sudanese female refugees in Uganda: a cluster randomised trial. Lancet Glob Health 2020;8:e254-63.

73 Weine S, Kulauzovic Y, Klebic A, et al. Evaluating a multiple-family group access intervention for refugees with PTSD. J Marital Fam Ther 2008;34:149-64.

74 Yurtsever A, Konuk E, Akyüz T, et al. An eye movement desensitization and reprocessing (EMDR) group intervention for Syrian refugees with post-traumatic stress symptoms: results of a randomized controlled trial. Front Psychol 2018;9:493.

75 Tonin FS, Rotta I, Mendes AM, et al. Network meta-analysis: a technique to gather evidence from direct and indirect comparisons. Pharm Pract 2017;15:943.

76 Purgato M, Cuijpers P, Barbui C. Regulatory requirements for psychological interventions. Lancet Psychiatry 2021;8:174-6.

77 Byrow Y, Pajak R, Specker P, et al. Perceptions of mental health and perceived barriers to mental health help-seeking amongst refugees: a systematic review. Clin Psychol Rev 2020;75:101812.

78 Due C, Green E, Ziersch A. Psychological trauma and access to primary healthcare for people from refugee and asylum-seeker backgrounds: a mixed methods systematic review. Int J Ment Health Syst 2020;14:71.

79 Nosè M, Turrini G, Barbui C. Access to mental health services and psychotropic drug use in refugees and asylum seekers hosted in high-income countries. Epidemiol Psychiatr Sci 2015;24:379-81.

80 Sijbrandij M. Expanding the evidence: key priorities for research on mental health interventions for refugees in high-income countries. Epidemiol Psychiatr Sci 2018;27:105-8.
81 Karatzias T, Murphy P, Cloitre M, et al. Psychological interventions for ICD-11 complex PTSD symptoms: systematic review and metaanalysis. Psychol Med 2019;49:1761-75.

82 Wampold BE. How important are the common factors in psychotherapy? an update. World Psychiatry 2015;14:270-7.

83 Clay J, Eaton J, Gronholm PC, et al. Core components of mental health stigma reduction interventions in low- and middleincome countries: a systematic review. Epidemiol Psychiatr Sci 2020;29:e164.

84 Giacco D, Priebe S. Mental health care for adult refugees in highincome countries. Epidemiol Psychiatr Sci 2018;27:109-16.

85 Bryant RA, Schafer A, Dawson KS, et al. Effectiveness of a brief behavioural intervention on psychological distress among women with a history of gender-based violence in urban Kenya: a randomised clinical trial. PLoS Med 2017;14:e1002371.

86 de Graaff AM, Cuijpers P, Acarturk C, et al. Effectiveness of a peerrefugee delivered psychological intervention to reduce psychological distress among adult Syrian refugees in the Netherlands: study protocol. Eur J Psychotraumatol 2020;11:1694347.

87 Purgato M, Carswell K, Acarturk C, et al. Effectiveness and cost-effectiveness of self-help plus $(\mathrm{SH}+)$ for preventing mental disorders in refugees and asylum seekers in Europe and turkey: study protocols for two randomised controlled trials. BMJ Open 2019;9:e030259.

88 Rahman A, Riaz N, Dawson KS, et al. Problem management plus $(\mathrm{PM}+)$ : pilot trial of a who transdiagnostic psychological intervention in conflict-affected Pakistan. World Psychiatry 2016;15:182-3.

89 Sangraula M, Turner EL, Luitel NP. Feasibility of group problem management plus $(\mathrm{PM}+)$ to improve mental health and functioning of adults in earthquake-affected communities in Nepal. Epidemiol Psychiatr Sci 2020;29:e130.

90 Barbui C, Purgato M, Abdulmalik J, et al. Efficacy of psychosocial interventions for mental health outcomes in low-income and middle-income countries: an umbrella review. Lancet Psychiatry 2020;7:162-72

91 Hamdani SU, Huma Z-E-, Rahman A, et al. Cost-Effectiveness of who problem management plus for adults with mood and anxiety disorders in a post-conflict area of Pakistan: randomised controlled trial. Br J Psychiatry 2020;217:623-9.

92 Giacco D, Matanov A, Priebe S. Providing mental healthcare to immigrants: current challenges and new strategies. Curr Opin Psychiatry 2014:27:282-8.

93 Gillespie M, Ampofo L, Cheesman M. Mapping refugee media journeys Smartphones and social media networks, 2016. Available: https://www.statewatch.org/media/documents/news/2016/may/oumapping-refugee-media-journeys.pdf [Accessed 11 Sep 2020].

94 Etzelmueller A, Vis C, Karyotaki E, et al. Effects of Internet-based cognitive behavioral therapy in routine care for adults in treatment for depression and anxiety: systematic review and meta-analysis. $J$ Med Internet Res 2020;22:e18100.

95 Jones C, Miguel-Cruz A, Smith-MacDonald L, et al. Virtual TraumaFocused therapy for military members, Veterans, and public safety personnel with posttraumatic stress injury: systematic scoping review. JMIR Mhealth Uhealth 2020;8:e22079.

96 Bjertrup PJ, Bouhenia M, Mayaud P, et al. A life in waiting: refugees' mental health and narratives of social suffering after European Union border closures in March 2016. Soc Sci Med 2018;215:53-60. 